\title{
Apoptosis-based therapy to treat pulmonary arterial hypertension
}

Yuichiro J. Suzuki ${ }^{1}$, Yasmine F. Ibrahim ${ }^{1,2}$ and Nataliia V. Shults ${ }^{1 *}$

Department of Pharmacology and Physiology, Georgetown University Medical Center, Washington DC 20007 USA ${ }^{2}$ Department of Pharmacology, Minia University School of Medicine, Minia, Egypt

Article Info

\section{Article Notes}

Received: July 27, 2016

Accepted: August 27, 2016

*Correspondence:

Nataliia V. Shults, Department of Pharmacology and Physiology,

Georgetown University Medical Center, Washington DC 20007

USA

E-mail: ns1015@georgetown.edu

C 2016 Nataliia V. Shults. This article is distributed under the terms of the Creative Commons Attribution 4.0 International License.

\section{Keywords}

Anti-tumor drugs

Apoptosis

Cancer chemotherapeutic agents

Pulmonary arterial hypertension

Pulmonary hypertension

Right heart failure

Right ventricle

Vascular remodeling

\section{ABSTRACT}

Pulmonary arterial hypertension $(\mathrm{PAH})$ is rare, but patients who are diagnosed with this disease still suffer from a lack of satisfactory treatment strategies to prolong survival. While currently approved drugs for PAH have some benefits, these vasodilators only have limited efficacy for eliminating pulmonary vascular remodeling and reducing mortality. Thus, our laboratory has been exploring the use of aggressive drugs, which are capable of causing apoptotic cell death, to treat $\mathrm{PAH}$. We have so far found that three classes of anti-tumor agents, including anthracyclines, taxanes, and proteasome inhibitors, are capable of reducing pulmonary vascular thickness in rats with $\mathrm{PAH}$. These drugs kill cells in remodeled pulmonary vessels without affecting the normal, healthy pulmonary vasculature, revealing that proliferating vascular cells in $\mathrm{PAH}$ patients are more sensitive to drug-induced apoptosis compared to the differentiated phenotype that is physiologically important for smooth muscle contraction. Since many apoptosis-inducing drugs cause cardiotoxicity in cancer patients, and because $\mathrm{PAH}$ patients already have a weakened heart, we focus on finding biological mechanisms that may reverse pulmonary vascular remodeling without promoting cardiotoxicity. We found two agents, dexrazoxane and pifithrin- $\alpha$, that selectively inhibit cardiac muscle apoptosis without affecting the drug-induced apoptosis of the proliferating pulmonary vascular cells. Thus, we propose that the addition of apoptosis-inducing drugs and cardioprotectants to $\mathrm{PAH}$ therapies may be effective in treating patients and preventing right heart failure.

\section{Introduction}

Pulmonary arterial hypertension (PAH) is a rare disease with prevalence estimated between 15 and 50 cases per million people ${ }^{1}$. $\mathrm{PAH}$ prevalence is two-to-four times higher in women than in men $^{2,3}$, and the disease can affect people of any age, even children. The early symptoms of PAH include mild dyspnea, fatigue, and dizziness, which can also be associated with various other diseases. Further, accurate diagnosis of PAH requires an invasive right-heart catheterization ${ }^{2}$. As a result, $\mathrm{PAH}$ diagnosis is often delayed and, by the time patients are diagnosed with $\mathrm{PAH}$, the disease is progressed to the occurrence of pulmonary vascular remodeling and right ventricle $(\mathrm{RV})$ remodeling ${ }^{3}$.

In PAH, elevated pulmonary vascular resistance causes stress to the RV, and RV failure is the major cause of death in patients with $\mathrm{PAH}^{4}$. The narrowing and occlusion of pulmonary vessels, as well as the elevation of pulmonary vascular resistance are due to multiple factors, including vasoconstriction and the thickening of pulmonary vascular walls. Currently approved drugs for PAH treatment primarily cause vasodilation, but these have limited influence on the structural changes in the vessel walls. 
Increased pulmonary vascular wall thickness and the narrowing and, in some cases occlusion, of the pulmonary arterial lumens are due to the growth of pulmonary arterial cells such as smooth muscle cells, endothelial cells, and fibroblasts, which form various lesions including medial hypertrophy; eccentric intimal proliferation; concentric, non-laminar intimal thickening; concentric, laminar intimal thickening; fibrinoid necrosis; occlusive intimal proliferation; and plexiform legions. Figure 1 exhibits some pulmonary vascular lesions in PAH with narrow or occluded blood vessel lumens that would significantly limit blood flow. Thus, therapeutic strategies to inhibit cell-
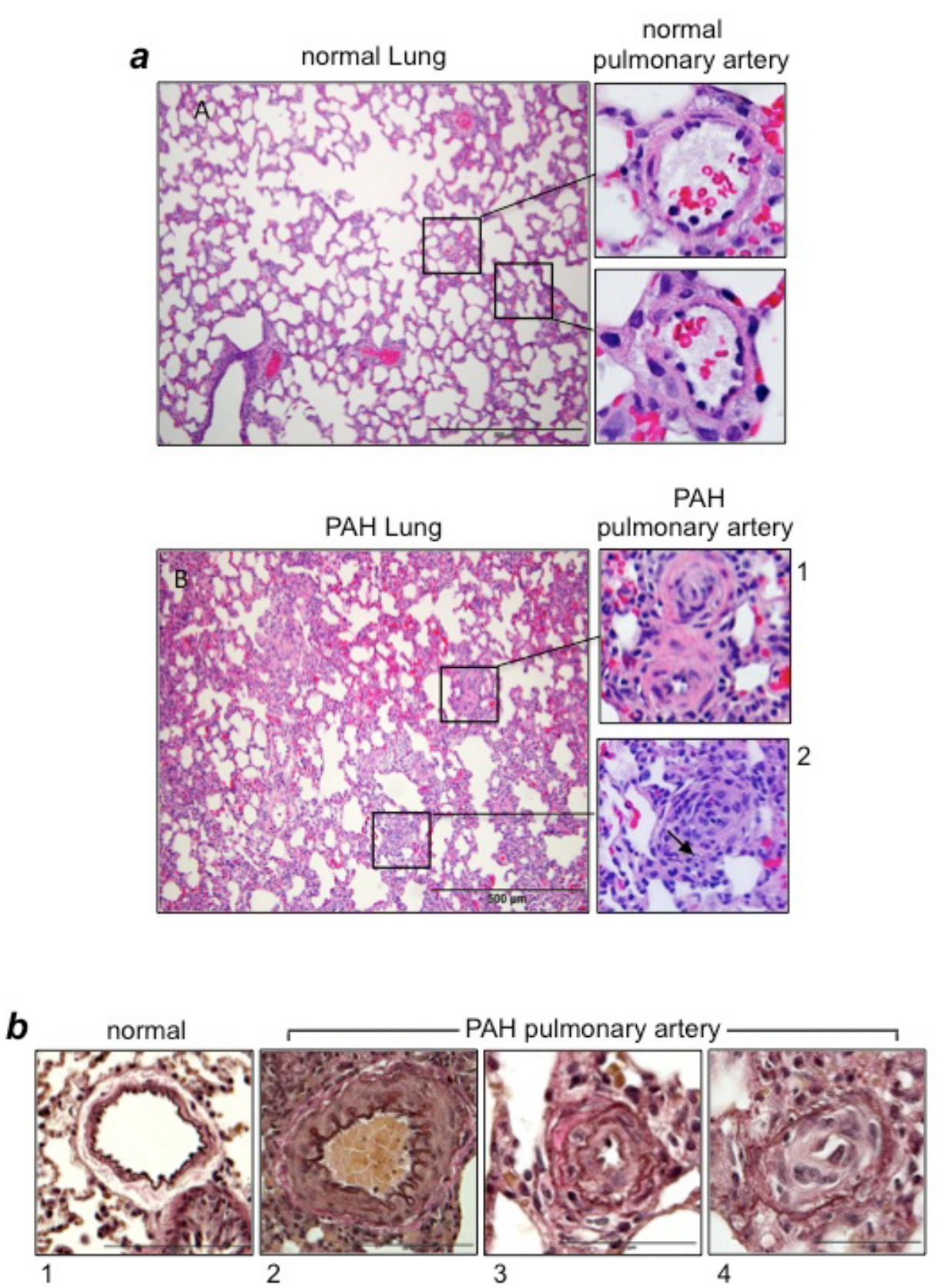

Figure 1: Vascular lesions in PAH.

(a) Hematoxylin and eosin (H\&E) stain of the lung and pulmonary artery (PA) from normal control rats and rats with PAH induced by SU5416/hypoxia ${ }^{12,20}$. In PAH lungs, atelectasis and emphysematous expansion of alveoli with thickened alveolar septa are seen. Around the vessels, perivascular inflammatory infiltrates are present. Panel 1 shows occluded small PAs in rats with PAH. Panel 2 shows the occluded central artery and a rounded collection of endothelial cells forming small channels (arrow). (b) Verhoeff-Van Gieson stains of PAs. Panel 1 shows thin intima and muscular media in normal control rats. By contrast, PAs of rats with PAH induced by SU5416/hypoxiaa ${ }^{12,20}$ exhibit hypertrophy and hyperplasia of medial smooth muscle cells and an increased amount of collagen fibers (Panel 2); the intimal thickening due to endothelial cell proliferation and the destruction of the inner elastic lamina (Panel 3), and intimal proliferation characterized by onion-skin-like layers with narrow lumens and no shirring of elastic membranes (Panel 4). 
growth signaling should prevent the formation and delay the progression of pulmonary vascular remodeling.

However, as noted above, PAH patients are often diagnosed after developing severe changes in the pulmonary vasculature, suggesting that inhibiting cell growth may have only limited effects in terms of reducing vascular resistance. Thus, our laboratory is exploring the effects of therapeutic agents that are capable of causing the death of pulmonary vascular cells in order to eliminate lesions that contribute to the narrowing and occlusion of the pulmonary vessels (Figure 1). Since it is preferable to kill unwanted cells in a controlled fashion, inducing programmed cell death, such as apoptosis, may be a fruitful avenue. Though, we refer to this approach as "apoptosisbased therapy," the mechanisms of programmed cell death could include those other than apoptosis, as discussed below.

Research in cancer therapy, which also frequently utilizes apoptosis-based approach, has demonstrated that one major problem with using cell death-causing agents is that they can also cause the deaths of cells which we do not want to kill. Notably, many anti-tumor drugs cause the death of cardiac myocytes, exerting cardiotoxicity ${ }^{5}$. Since $\mathrm{PAH}$ patients already suffer from RV dysfunctions, the use of such drugs, which could potentially worsen the cardiac problems in PAH patients, poses challenges. In this regard, over the last decade, our laboratory has been investigating: (i) whether pulmonary vascular cells are susceptible to apoptosis; (ii) whether the administration of apoptosisinducing, anti-tumor drugs reverses pulmonary vascular remodeling; and (iii) what the effects of these drugs are on RVs influenced by PAH.

\section{Effects of Anti-Tumor Drugs on Pulmonary Vascular Remodeling}

In considering which anti-tumor agents should be first studied in order to develop apoptosis-based therapy for PAH, we decided to use daunorubicin (DNR). DNR is the first member of the anthracycline class of anti-tumor agents and has been used clinically for 50 years. One reason we investigated this drug is that DNR was found to be an effective inducer of apoptosis in cultured human pulmonary artery smooth muscle cells (PASMCs) ${ }^{6}$. Another important reason was that since anthracyclines have cardiotoxic properties, particularly left ventricle (LV) toxicity, in cancer patients ${ }^{5}$, such drugs may serve as an excellent model for understanding how normal RVs and RVs affected by PAH may be influenced by anti-tumor drugs. While the RV and LV are generally thought to have identical molecular characteristics, our studies have revealed differential molecular-signaling mechanisms in the two ventricles ${ }^{7,8}$. Thus, we thought we might find that the RV is not as susceptible as the LV to the toxic effects of anthracycline administration. Further, our studies using cultured cells revealed that the mechanisms of DNR-induced cell death processes differ between PASMCs and cardiac muscle cells ${ }^{9}$. We reported that DNR kills cardiac muscle cells via p53-dependent inhibition of the DNA-binding activity of the CBF/NF-Y transcription factor toward the CCAAT box within the promoter region of Gata4 that regulates the gene transcription of anti-apoptotic proteins ${ }^{10}$. In cardiac muscle cells, a p53 inhibitor, pifithrin- $\alpha$, suppresses DNR-induced CBF/NF-Y activity and therefore GATA4 downregulation and cell death (Figure 2a). By contrast, in PASMCs, while DNR did inhibit GATA4 and CBF/NF-Y DNA-binding activities, the inhibition of p53 by pifithrin- $\alpha$ had no effects (Figure 2b). These results suggest that p53 inhibitors protect the heart without influencing the ability of DNR to kill PASMCs.

To determine the effect of DNR in intactanimals, SpragueDawley rats were exposed to chronic hypoxia for 2 weeks to develop pulmonary hypertension and pulmonary vascular remodeling, characterized by thickening of the smooth muscle mass. After pulmonary hypertension and vascular thickening were developed, DNR was administered. We found that, within 3 days, DNR at a concentration as low as $5-\mathrm{mg} / \mathrm{kg}$ body weight significantly reduced pulmonary arterial wall thickness, a reduction associated with
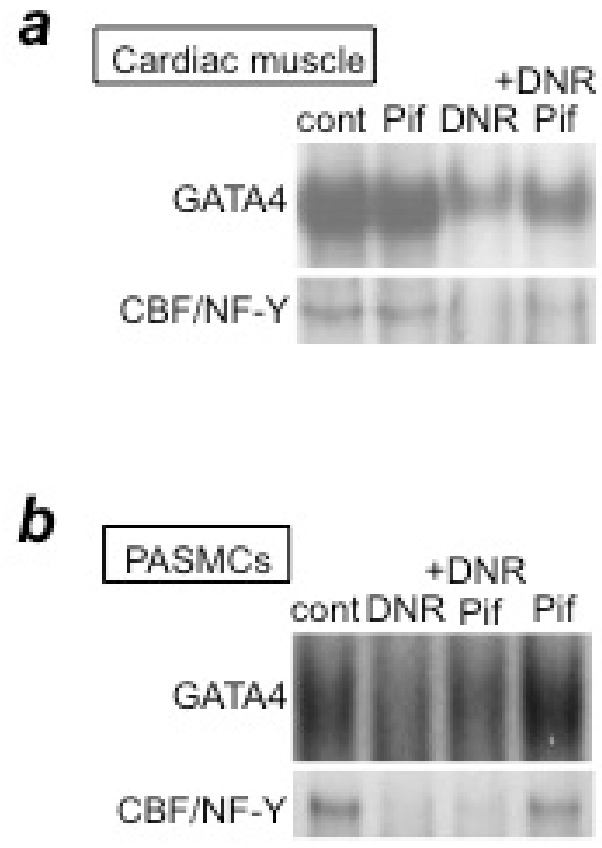

Figure 2: p53 inhibition by pifithrin- $\alpha$ blocks DNR-induced downregulation of GATA4 and CBF/NF-Y in cardiac muscle cells, but not in PASMCs.

(a) Cardiac muscle cells or (b) PASMCs were pre-treated with pifithrin- $\alpha$ (Pif) and then treated with DNR. Nuclear extracts were prepared and the DNA-binding activities of GATA4 and CBF/NF-Y were monitored by electrophoretic mobility shift assays. 
increased apoptosis ${ }^{11,12}$. Importantly, neither the reduction of vascular wall thickness nor the induction of apoptosis occurred in the pulmonary vessels of normal control rats, suggesting that remodeled pulmonary vasculature is sensitive to DNR-induced cell killing.

We found that proteasome inhibitors -- bortezomib and MG-132 -- also reversed pulmonary vascular remodeling in chronic hypoxia-induced pulmonary hypertensive rats ${ }^{12,13}$. Bortezomib has been used to treat multiple myeloma and relapsed mantle cell lymphoma. ${ }^{14}$ To produce the more severe pulmonary vascular remodeling, resembling the human disease, rats were injected with SU5416 (inhibitor of the vascular endothelial growth factor receptor tyrosine kinase) and then subjected to chronic hypoxia for 3 weeks ${ }^{12,15,16}$. Subsequently, maintaining these rats in normoxia for 2 weeks developed medial as well as intimal thickening in the pulmonary vasculature, and proteasome inhibition effectively reduced vascular wall thickness in this model ${ }^{12}$.

\section{Possible Mechanism for the Increased Susceptibility} of Remodeled Pulmonary Vasculature to Cell Killing

As described above, DNR reduced the thickness of remodeled pulmonary arterial walls in rats exposed to
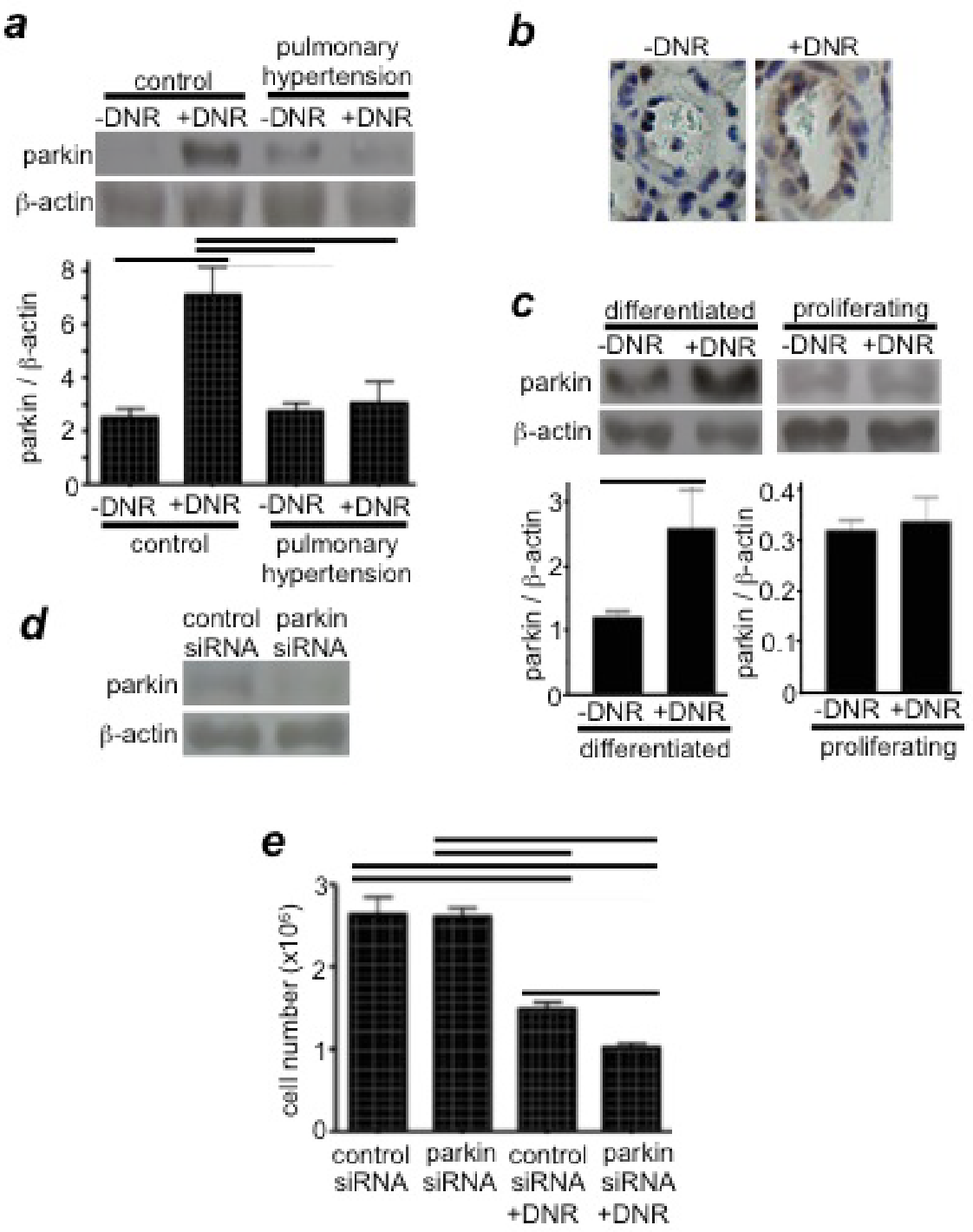

Figure 3: Parkin regulates drug-induced cell death.

(a) Rats were exposed to hypoxia for two weeks to develop pulmonary hypertension, and were then injected with DNR ( $5 \mathrm{mg} / \mathrm{kg}$ body weight). Rats were then placed back in hypoxia. One day after the injection, isolated pulmonary arteries were homogenized, and parkin levels were monitored by Western blotting. Reproduced from Ibrahim et al. ${ }^{12}$ (b) Parkin expression levels were monitored by immunohistochemistry using the parkin antibody. (c) Differentiated and proliferating cultured human PASMCs were treated with DNR for $5 \mathrm{~h}$. Cell lysates were prepared, and parkin levels were monitored by Western blotting. Reproduced from Ibrahim et al. ${ }^{12}(\mathrm{~d})$ The extent of parkin knockdown by transfecting human PASMCs with control or parkin siRNA. (e) Human PASMCs transfected with control or parkin siRNA were treated with DNR, and the cell number was determined by counting on a hemocytometer. Reproduced from Ibrahim et al. ${ }^{12}$ Bar graphs represent means \pm SEM. Bars denote the values significantly different from each other at $\mathrm{P}<0.05$. 
chronic hypoxia without affecting the pulmonary vessels of normoxic control rats. Understanding this remarkable mechanism should help in developing improved therapeutic agents for PAH.

Our experiments suggested that parkin, an E3 ubiquitin ligase that plays an important role in Parkinson's disease ${ }^{17}$, is also expressed in PASMCs and may define the mechanism for the increased susceptibility of remodeled pulmonary vascular walls to DNR-induced cell death. We found that DNR increases the expression of parkin in the pulmonary arteries of normal rats, but not in rats with PAH (Figures $3 \mathrm{a} \& 3 \mathrm{~b})^{12}$. Similarly, DNR only increased parkin expression in cultured human PASMCs that had been differentiated to have the contractile phenotype, but not in proliferating cells (Figure 3c) ${ }^{12}$. Thus, in normal contractile PASMCs, parkin may suppress DNR-induced cell killing. Indeed, parkin seems to function as a cell survival factor in PASMCs, as siRNA knockdown of parkin exaggerated DNR-induced cell killing (Figures $3 \mathrm{~d} \& 3 \mathrm{e})^{12}$. Future investigations along this line should shed light on an approach to most effectively target unwanted remodeled pulmonary vascular cells.

\section{Anti-Tumor Drugs and Cardiotoxicity in PAH}

Our study noted no apparent cardiotoxicity, such as the induction of apoptosis by DNR ( $5 \mathrm{mg} / \mathrm{kg}$ body weight), in the hypertrophied $\mathrm{RV}^{12}$. However, since bortezomib was reported to produce cardiotoxicity in animals with pulmonary hypertension ${ }^{18}$, we explored the effects of carfilzomib (CFZ), considered to be a safe and effective alternative to bortezomib in the treatment of multiple myeloma $^{19}$. In two rat models of PAH (SU5416/hypoxia and SU5416/ovalbumin), CFZ reduced pulmonary vascular wall thickness and induced programmed cell death (Figure $4)^{20}$. Like DNR, the effects of CFZ were only observed in the pulmonary vasculatures of rats with $\mathrm{PAH}$, but not in normal control rats. While the remodeled RVs were not susceptible to CFZ-induced apoptosis, significant apoptosis was observed in the LVs of animals with PAH. To find possible cardioprotective agents, in accordance with the cell-culture experiments described above, pifithrin- $\alpha$ (a p53 inhibitor) was administered along with CFZ in rats with PAH. Remarkably, pifithrin- $\alpha$ completely protected the LV against CFZ-induced apoptosis. Moreover, pifithrin- $\alpha$ did not affect the action of CFZ to induce the apoptosis of pulmonary vascular cells or to reduce vascular wall thickness in rats with $\mathrm{PAH}^{20}$. Thus, p53 inhibitors protect the heart against drug-induced toxicity without altering the efficacy of antitumor agents to reverse pulmonary vascular remodeling. While we do not yet understand the mechanism, a clinically used, FDA-approved cardioprotectant against anti-tumor agents, dexrazoxane, also exhibited protective effects against CFZ-induced cardiotoxicity in PAH rats without affecting the efficacy of CFZ to reverse pulmonary vascular remodeling ${ }^{20}$. Thus, including dexrazoxane or pifithrin- $\alpha$
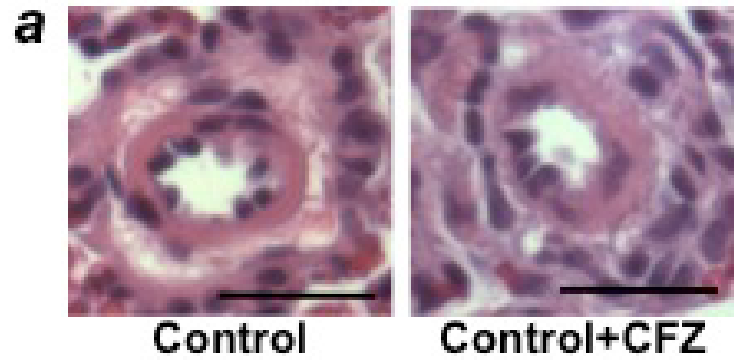

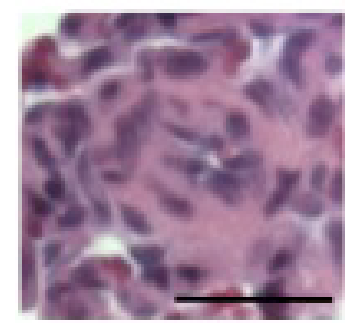

PAH

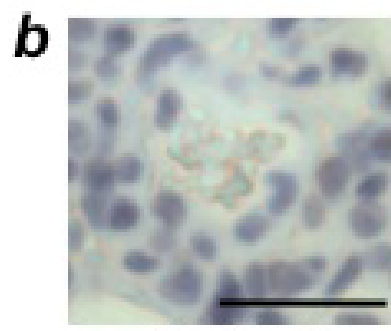

Control

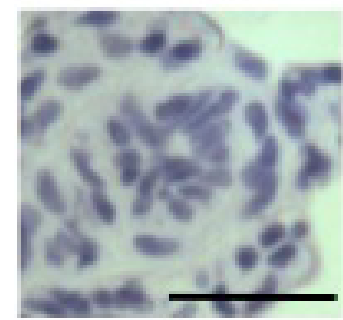

PAH

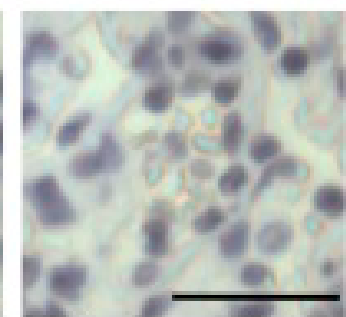

Control+CFZ

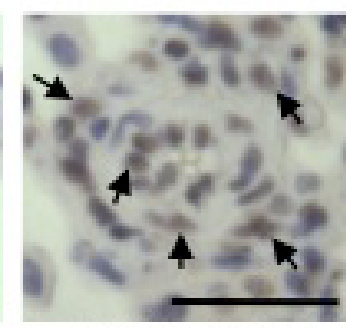

$\mathrm{PAH}+\mathrm{CFZ}$
Figure 4: CFZ reduces the wall thickness of remodeled pulmonary arteries and promotes apoptosis without affecting normal pulmonary arteries in SU5416/hypoxia model of PAH.

Rats were injected with SU5416 and exposed to hypoxia for 3 weeks, and then placed in normoxia for 5 weeks. Rats were then injected with $6 \mathrm{mg} / \mathrm{kg}$ body weight CFZ, twice per week for 2 weeks. The rats were sacrificed after 3 days of the last injection. (a) Representative $H \& E$ staining of small pulmonary arteries (diameters ranging from $50 \mu \mathrm{m}$ to $100 \mu \mathrm{m}$ ). Scale bars, 50 $\mu \mathrm{m}$. (b) Representative TUNEL assay results. Scale bars, $50 \mu \mathrm{m}$. Reproduced from Wang et al. ${ }^{20}$

should be considered during apoptosis-based therapy to treat PAH using anti-tumor drugs.

Targeting the RV Damage Promoted by PAH in the Treatment Strategy

Pulmonary hypertension per se does not kill patients; rather, right heart failure is the major cause of death among 
PAH patients. In addition to pulmonary vascular remodeling and severe narrowing and occlusion of the pulmonary vascular lumen, by the time patients are diagnosed with $\mathrm{PAH}$, severe RV modifications have already occurred, including the death of RV myocytes as well as the induction of RV fibrosis. Therefore, a treatment that can preferentially kill remodeled pulmonary vascular cells without affecting normal cells and also reduce mechanisms that contribute to these RV dysfunctions would be ideal. As shown in Figure $5 \mathrm{a}$, in response to $\mathrm{PAH}$, the RV suffers from major damage to the myocardium. H\&E stain shows the cardiomyocytes degeneration; polymorphic and hypertrophied cardiomyocytes; myofiber disarray; hypereosinophilia and hyperbasophilia of cardiomyocytes; contractures of cardiomyocytes (black arrow); myocytolysis (yellow arrow); a loss of myofiber striation; wavy arrangement of myofiber; edema of the cytoplasm; and indistinct nuclear borders within cardiomyocytes. A dramatic increase in the levels of collagen was also demonstrated in the RVs of PAH animals, as shown by the blue stain in Masson's trichrome-stained RV sections (Figure $5 \mathrm{~b}$ ). These changes in the RVs of PAH animals are also associated with the
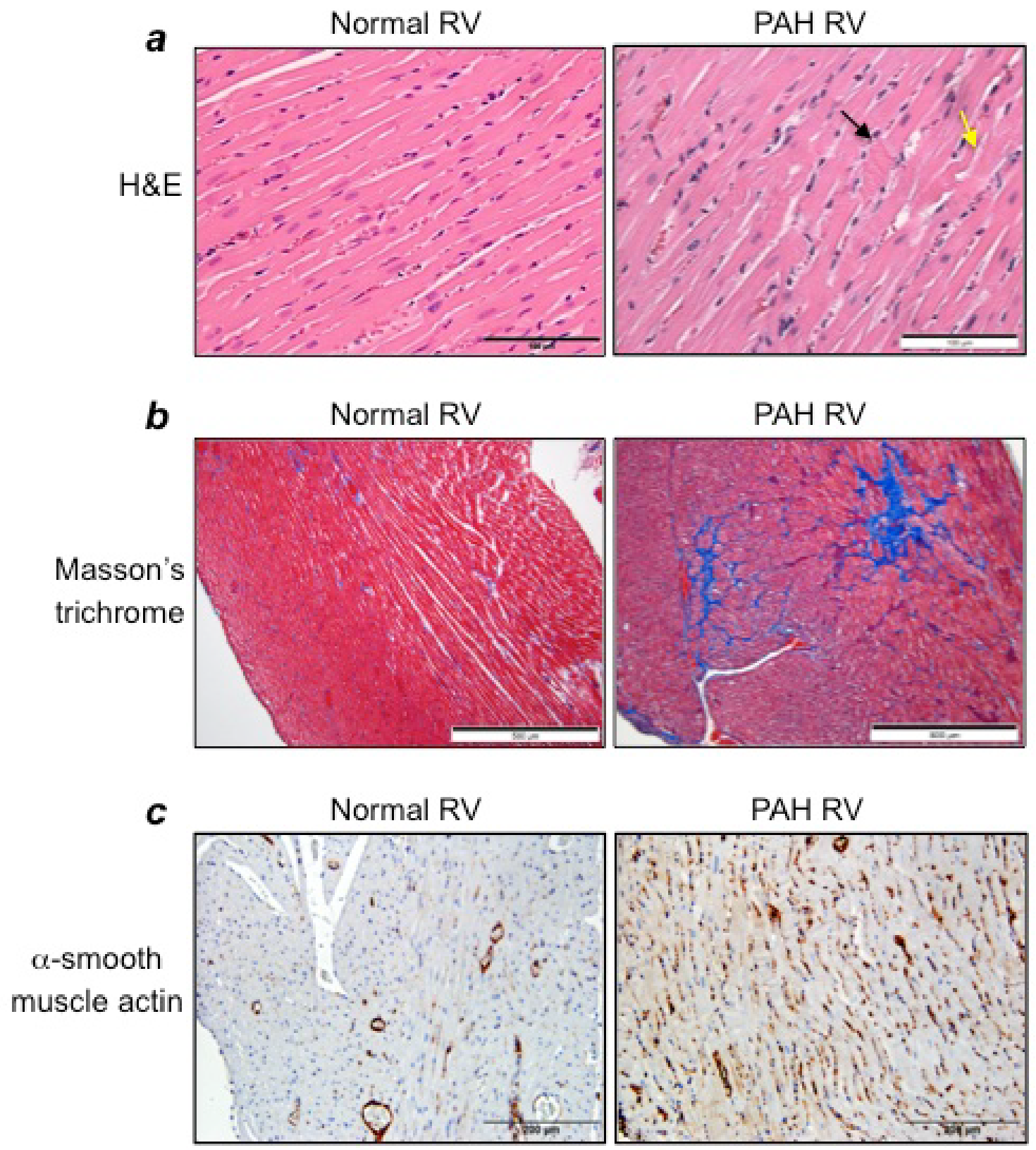

Figure 5: Characteristics of the RV in PAH.

Rats were subjected to SU5416/hypoxia treatment, and then hearts were fixed in $10 \%$ formalin and sectioned for histology analyses. (a) H\&E stain (Magnification x400), (b) Masson's trichrome staining (Magnification x100), (c) Immunohistochemistry with $\alpha$-smooth muscle actin antibody (Magnification x200). 
increased expression of $\alpha$-smooth muscle actin (Figure 5c), indicating the formation of myofibroblasts in the RVs of rats with PAH. Thus, the treatment strategy that can kill cardiac fibroblasts and/or myofibroblasts without affecting cardiomyocytes and activate collagen-degrading systems such as matrix metalloproteinases would be desirable. Subsequently, eliminated fibrotic areas need to be replaced by functional myocardium, perhaps through the formation of cardiomyocytes from resident cardiac progenitor cells. For example, isl1-positive cells that serve as progenitors of the secondary heart field from which the RV is derived during development ${ }^{21}$ may contribute to the restoration of RV myocytes. The inclusion of agents that can activate these mechanisms in the treatment strategies would help repairing the damaged RV in PAH patients.

\section{Summary and Future Perspectives}

Currently, no therapeutic agents are capable of satisfactorily prolonging the lives of patients who are diagnosed with $\mathrm{PAH}$, perhaps because currently available therapies have only limited effects on the pulmonary vascular remodeling that is an important aspect of this disease. Histologic observations in humans and experimental animals show dramatic narrowing and occlusion of the pulmonary vascular lumens, clearly indicating limited blood flow. Thus therapeutic strategies to reverse the narrowing and occlusion of the pulmonary vascular lumen should be necessary aspects of the treatment of PAH. In rat models of PAH, our laboratory observed that some cell-death-inducing anti-tumor drugs reverse pulmonary vascular remodeling without influencing the normal pulmonary vasculature. Further, the perception that giving these drugs to PAH patients is contraindicated appears to be incorrect, as remodeled RVs were found to be more resistant to damage by these drugs, and we discovered agents that are capable of protecting the heart without reducing the anti-tumor drug's efficacy in the pulmonary vasculature. Collectively, these data generated using animal models of PAH suggest that combining cellkilling drugs and cardioprotectants with the currently available vasodilator therapies may present optimal therapeutic strategies to treat this lethal disease. Clinical studies to test this concept are warranted. Successfully curing PAH, however, depends on future studies that use experimental models to define exactly which anti-tumor agents (either alone or in combination) should be given via which route of administration, which doses, and under which timing or duration of treatment. Further, possible unwanted effects of such treatment regimes, such as drug resistance, should also be investigated.

\section{Acknowledgements}

This work was supported in part by National Institutes of Health (R01HL72844 and R03AG047824) to YJS. The content is solely the responsibility of the authors and does not necessarily represent the official views of the National Institutes of Health.

\section{References}

1. Peacock AJ, Murphy NF, McMurray JJ, Caballero L, Stewart S. An epidemiological study of pulmonary arterial hypertension. Eur Respir J. 2007; 30:104-109.

2. Gaine SP, Rubin LJ. Primary pulmonary hypertension. Lancet. 1998; 352:719-725.

3. Badesch DB, Raskob GE, Elliott CG, et al. Pulmonary arterial hypertension: baseline characteristics from the REVEAL Registry. Chest. 2010; 137:376-387.

4. Voelkel NF, Quaife RA, Leinwand LA, Barst RJ, McGoon MD, Meldrum DR, et al. National Heart, Lung, and Blood Institute Working Group on Cellular and Molecular Mechanisms of Right Heart Failure. Right ventricular function and failure: report of a National Heart, Lung, and Blood Institute working group on cellular and molecular mechanisms of right heart failure. Circulation. 2006; 114:1883-1191.

5. Vincent DT, Ibrahim YF, Espey MG, Suzuki YJ. The role of antioxidants in the era of cardio-oncology. Cancer Chemother Pharmacol. 2013; 72:1157-1168.

6. Suzuki YJ, Nagase H, Wong CM, Kumar SV, Jain V, Park AM, et al. Regulation of Bcl-xL expression in lung vascular smooth muscle. Am J Respir Cell Mol Biol. 2007; 36:678-687.

7. Liu L, Marcocci L, Wong CM, Park AM, Suzuki YJ. Serotonin-mediated protein carbonylation in the right heart. Free Radic Biol Med. 2008; 45:847-854.

8. Park AM, Wong CM, Jelinkova L, Liu L, Nagase H, Suzuki YJ. Pulmonary hypertension-induced GATA4 activation in the right ventricle. Hypertension. 2010; 56:1145-1151.

9. Suzuki YJ, Liu L, Park A. Differential mechanisms of apoptosis in pulmonary artery smooth muscle cells and in cardiac muscle cells. Am J Respir Crit Care Med. 2009; 179:A5359.

10. Park AM, Nagase H, Liu L, Vinod Kumar S, Szwergold N, Suzuki YJ, et al. Mechanism of anthracycline-mediated down-regulation of GATA4 in the heart. Cardiovasc Res. 2011; 90:97-104.

11. Jelinkova L, Suzuki YJ. Effects of daunorubicin on pulmonary artery smooth muscle cells in rat model of hypoxia-induced pulmonary hypertension. Am J Resp Crit Care Med. 2010; 181:A1181.

12. Ibrahim YF, Wong CM, Pavlickova L, Liu L, Trasar L, Bansal G, et al. Mechanism of the susceptibility of remodeled pulmonary vessels to drug-induced cell killing. J Am Heart Assoc. 2014; 3:e000520.

13. Wong CM, Suzuki YJ. Proteasome inhibitors reverse pulmonary vascular remodeling. Am J Respir Crit Care Med. 2012; 185:A2621.

14. Chen D, Frezza M, Schmitt S, Kanwar J, Dou QP. Bortezomib as the first proteasome inhibitor anticancer drug: current status and future perspectives. Curr Cancer Drug Targets. 2011; 11:239-253.

15. Taraseviciene-Stewart L, Kasahara Y, Alger L, Hirth P, Mc Mahon G, Waltenberger J, et al. Inhibition of the VEGF receptor 2 combined with chronic hypoxia causes cell death-dependent pulmonary endothelial cell proliferation and severe pulmonary hypertension. FASEB J. 2001; 15:427-438.

16. Oka M, Homma N, Taraseviciene-Stewart L, Morris KG, Kraskauskas D, Burns N, et al. Rho kinase-mediated vasoconstriction is important in severe occlusive pulmonary arterial hypertension in rats. Circ Res. 2007; 100:923-929.

17. Caulfield TR, Fiesel FC, Springer W. Activation of the E3 ubiquitin ligase Parkin. Biochem Soc Trans. 2015; 43:269-274. 
18. Kim SY, Lee JH, Huh JW, Kim HJ, Park MK, Ro JY, et al. Bortezomib alleviates experimental pulmonary arterial hypertension. Am J Respir Cell Mol Biol. 2012; 47:698-708.

19. McBride A, Klaus JO, Stockerl-Goldstein K. Carfilzomib: a secondgeneration proteasome inhibitor for the treatment of multiple myeloma. Am J Health Syst Pharm. 2015; 72:353-360.
20. Wang X, Ibrahim YF, Das D, Zungu-Edmondson M, Shults NV, Suzuki YJ. Carfilzomib reverses pulmonary arterial hypertension. Cardiovasc Res. 2016; 110:188-199.

21. Iancu CB, Iancu D, Renţea I, Hostiuc S, Dermengiu D, Rusu MC. Molecular signatures of cardiac stem cells. Rom J Morphol Embryol. $2015 ; 56: 1255-1262$. 